\title{
Perdre ses lettres en république décadente : d'une poétique de l'internat dans Le Rivage des Syrtes de Julien Gracq
}

\section{Émile Bordeleau-Pitre}

\section{(2) OpenEdition \\ Journals}

Édition électronique

URL : http://journals.openedition.org/pratiques/7306

DOI : $10.4000 /$ pratiques. 7306

ISSN : 2425-2042

Éditeur

Centre de recherche sur les médiations (CREM)

\section{Référence électronique}

Émile Bordeleau-Pitre, «Perdre ses lettres en république décadente : d'une poétique de l'internat dans Le Rivage des Syrtes de Julien Gracq », Pratiques [En ligne], 183-184 | 2019, mis en ligne le 30 décembre 2019, consulté le 11 octobre 2020. URL : http://journals.openedition.org/pratiques/7306 ; DOI : https://doi.org/10.4000/pratiques.7306

Ce document a été généré automatiquement le 11 octobre 2020.

(c) Tous droits réservés 


\title{
Perdre ses lettres en république décadente : d'une poétique de l'internat dans Le Rivage des Syrtes de Julien Gracq
}

\author{
Émile Bordeleau-Pitre
}

«Si peu de goût qu'on ait de proposer, en guise d'explication, des métaphores, la civilisation peut être comparée sans trop d'inexactitude à la mince

couche verdâtre - magma vivant et détritus variés - qui se forme à la surface des eaux calmes et se solidifie en croûte, jusqu'à ce qu'un remous

soit venu tout bouleverser. »

Michel Leiris, « Civilisation » dans Documents,

1929

« Un livre naît d'une insatisfaction, d'un vide dont les contours ne se révéleront précis qu'au cours du travail, et qui demande à être comblé par l'écriture », explique J. Gracq à J. Roudault dans un entretien (Gracq, 2002 [1981], p. 49). Longtemps après les œuvres de fiction l'ayant rendu célèbre (Au Château d'Argol en 1938; Le Rivage des Syrtes en 1951; Un Balcon en forêt en 1958), J. Gracq partage dans l'un de ses rares écrits autobiographiques, La Forme d'une ville, une expérience l'ayant profondément bouleversé et qui pourrait préciser la nature spécifique de cette "insatisfaction » propre, de ce "vide» intime demandant à être comblé : son passage par l'internat. J. Gracq fréquente effectivement dès 1921 le lycée Clemenceau à Nantes et vit le régime de l'internat jusqu'en 1935 (après Clemenceau à Henri-IV, puis à l'École normale supérieure et à Saint-Maixent). Cette expérience marque, de son propre aveu, l'une des principales cassures de sa vie (Gracq, 1989a, p. LXIII) ; «[t]out lui pèse, lui semble odieux : l'éloignement de la maison familiale, l'anonymat grisâtre des lieux, la promiscuité continue, la nourriture médiocre, la claustration, le caporalisme disciplinaire, la complète monotonie répétitive des journées » (ibid.). 
2 Traitant de cette expérience de l'internat, La Forme d'une ville fait aussi part des binarités, des dichotomies fondant le travail de l'imaginaire gracquien - son fonctionnement-, ce qui nous force à reconsidérer à leur aune toute la production ayant précédé leur exposition. Cependant, il faut préciser que ce n'était pas la première fois que J. Gracq témoignait de l'impact de l'expérience de l'internat sur l'écriture. Une vingtaine d'années plus tôt, il associait déjà l'esprit de révolte chez A. Rimbaud, Comte de Lautréamont et $\mathrm{A}$. Jarry à ce type particulier de formation, où claustration côtoie éducation militarisée : "Cette précocité qui leur est commune à l'âge où l'on quitte à peine les bancs du lycée n'est pas un pur effet du hasard: la société fixe elle-même à vingt ans, par ses méthodes de claustration absurdes, le moment de parler pour ceux qui ont survécu au dressage - de porter témoignage dans un cri avant qu'il ne soit trop tard » (Gracq, 1989b [1961], p. 895). Remarquons d'entrée de jeu l'opposition qui se fait jour très tôt chez J. Gracq, entre l'expression orale (« le moment de parler ») ou le cri (le bruit, ce qui de facto ne pourrait s'inscrire dans le texte mais dont on comprend qu'il le fait quand même puisqu'il est question ici de trois écrivains) et le «dressage » des internes: nous y reviendrons. De façon similaire dans La Forme d'une ville, le récit devient ce qui s'écrit contre les murs de l'internat; il garde, à ce titre, la marque de l'insurrection que J. Gracq lisait chez A. Rimbaud, Comte de Lautréamont et A. Jarry ; et l'extérieur, la liberté, ne se manifeste qu'à travers "la rumeur de Nantes » - c'est-àdire, encore une fois, qu'à travers le bruit.

Le régime de l'internat, dans les années vingt de ce siècle, était strict. Aucune sortie, en dehors des vacances, que celles du dimanche; encore fallait-il qu'un correspondant vînt prendre livraison de nous en personne au parloir, et, en principe, nous y ramener le soir. Je ne sortais qu'une fois par quinzaine; le reste du temps, je n'apercevais de la ville que la cime des magnolias du Jardin des plantes, par-dessus le mur de la cour, et la brève échappée sur la façade du musée que nous dévoilait le portail des externes, quand on l'ouvrait pour leur entrée, à huit heures moins cinq et à deux heures moins cinq. Mais cette réclusion si stricte était à sens unique. Deux fois par jour, comme la marée, avec le flot des externes, la rumeur de Nantes parvenait jusqu'à nous, tantôt filtrée, tantôt orchestrée. Je vivais au cœur d'une ville presque davantage imaginée que connue, où je possédais quelques repères solides, où certains itinéraires m'étaient familiers, mais dont la substance, l'odeur même, gardait quelque chose d'exotique: une ville où toutes les perspectives donnaient d'elles-mêmes sur des lointains mal définis, non explorés, un canevas sans rigidité, perméable plus qu'un autre à la fiction. Chacun des rhumbs qui étoilaient cette rose des vents fleurissait naturellement, indéfiniment, pour l'imagination. (Gracq, 1995 [1985], p. 772-773)

Ici, la réclusion de l'internat dans La Forme d'une ville devient non seulement l'une des prémisses à un récit autobiographique où Gracq raconte Nantes, qui pour lui reste cette "ville presque davantage imaginée que connue »; c'est aussi la claustration elle-même qui permet la construction d'un « canevas sans rigidité, perméable plus qu'un autre à la fiction ». Être enfermé - nous dit en quelque sorte J. Gracq-, ne pas pouvoir voir de l'autre côté des murs, nous oblige à imaginer ce qui se cache derrière, nous force, ultimement, à (se) raconter des histoires - et c'est là la seule transgression, la seule " révolte » envisageable. La transgression à travers la fiction manifeste donc une contradiction indépassable parce que structurelle: malgré le désir de sédition qui anime la plus grande part des fictions de J.Gracq, l'imagination, la mise en récit, l'écriture dépendent des murs contre lesquels elles s'érigent. Et c'est là tout le paradoxe qui compose son moteur d'écriture, sa " poétique de l'internat ", qui consiste à écrire contre les murs. Ici, Contre peut exprimer une opposition, dénoter une relation 
d'hostilité, de lutte dirigée envers les murs : ce qui est bel et bien le cas dans l'exemple du Rivage des Syrtes, comme nous le verrons, avec cette obsession omniprésente de la transgression qui caractérise le roman. Mais contre, lorsqu'on écrit contre les murs, peut nettement signifier le contraire objectif de cette ambition. En effet, l'expression peut désigner le support, la surface sur laquelle on écrit des histoires - ce qui tendrait à faire des murs, une condition nécessaire à l'écriture. Le désir tourne à vide, éteignant la possibilité de raconter, lorsqu'il obtient satisfaction. Qui plus est : la pratique même de l'écriture en est une qui se façonne et se polit à l'intérieur des murs de l'école, et donc de l'internat pour J. Gracq. S'insurger contre l'internat à travers un récit posé sur le papier ne manque donc pas d'ironie, dans la mesure où c'est ici avec les outils transmis par l'oppresseur qu'on tente de porter atteinte à l'oppression vécue.

\section{Les conformismes de l'écriture}

Même si le credo du XIX ${ }^{e}$ siècle sur la séquence scolarisation-alphabétisation - à l'aune de l'histoire qui a suivi - apparaît de plus en plus douteux si l'on en abstrait les autres conditions de la vie sociale (Furet \& Ozouf, 1997, p.11), il n'en reste pas moins que l'école reste l'endroit privilégié où l'on intègre les techniques propres aux cultures de l'écrit : la lecture, l'écriture et le calcul. Les grandes lois d'enseignement primaire ayant fondé le système scolaire français peuvent d'ailleurs en témoigner. Tant la Loi sur l'instruction primaire du 15 mars 1850 (Mayeur, 2004 [1981], p. 703) que la Loi du 28 mars 1882 sur l'enseignement primaire obligatoire (ibid., p. 711) insistent sur l'apprentissage obligatoire de ces compétences. La rédaction de cette législation entourant l'école répond à une nécessité neuve de combattre l'illettrisme pour former des citoyens qui seraient en mesure de répondre à des besoins autrefois absents.

Les contraintes de la société nouvelle vont se charger tout au cours du siècle de [généraliser l'instruction]. De plus en plus il devient indispensable d'être lettré pour lire, comprendre, signer les papiers administratifs, commerciaux, judiciaires, fiscaux, notariés dont le flot s'enfle, atteignant même les ruraux, même les pauvres. (Furet \& Ozouf, 1997, p. 150)

5 À cet égard, l'ambition est généralisée dans tous les camps: "Conservateurs et républicains s'accordent à valoriser hautement l'école comme instrument d'une alphabétisation que les uns et les autres proclament nécessaires » (ibid., pp. 9-10). Aller à l'école donc, depuis la propension exacerbée de construction d'écoles publiques après la Révolution française (propension qui inclut évidemment la construction des internats napoléoniens dont J. Gracq raconte l'expérience dans La Forme d'une ville), c'est nécessairement apprendre l'écriture.

Dans un univers social où la législation fait de l'écriture une obligation, l'internat devient donc ce lieu où l'apprentissage des techniques liées à la littératie constitue l'un des éléments fondamentaux du "dressage » auquel J. Gracq réfère dans son texte sur A. Rimbaud, Comte de Lautréamont et A. Jarry Parallèlement, les corps des internes se transforment en quelque sorte en " textes ", où s'écrivent les normes de comportement que doivent adopter les individus de même que les mesures de découragement des comportements jugés déviants - ce que M. Foucault (2013, p. 114) a qualifié de concept de "coercitif». Dans ces circonstances, faire œuvre d'insurrection, pour l'écrivain, s'attaquer aux murs de l'internat en d'autres mots, témoigne d'une aporie qui trouve difficilement résolution : écrire contre les murs se manifeste en effet par la mise à mal 
de l'écriture même, condition de possibilité de l'écrivain s'il en est une. Le Rivage des Syrtes représente ici un cas de figure paradigmatique, où l'homologie entre littératieoralité, orthodoxie-transgression et internat-imagination se fait extrêmement claire. Son analyse nous permet ainsi de mieux comprendre ce que nous pourrions qualifier de «poétique de l'internat».

7 Le Rivage des Syrtes met en scène la décadence d'une très vieille république, Orsenna, république fondée sur des hiérarchies où les textes administratifs et légaux sont de la plus haute importance. Orsenna est en guerre depuis trois siècles contre une nation ennemie, le Farghestan. Et si le texte est la pierre angulaire de la fondation et du maintien de la vieille république, les références au Farghestan, quant à elles, se construisent systématiquement dans Le Rivage des Syrtes autour de la notion de rumeur, d'incertitude et de bruit - c'est-à-dire autour de tout ce qui, par définition, ne saurait trouver place au sein d'un texte. Entre les deux nations ennemies se dresse une frontière jamais franchie, une "ligne continue d'un rouge vif", une "frontière d'alarme » (Gracq, 1989c [1951], p. 577). Le Rivage des Syrtes fait le récit d'une nation dangereuse mais fantasmée, société actualisée seulement par le désir, à partir d'une position où les conservatismes règnent en maîtres. On voit ici se déployer en creux, à travers la fiction, les circonstances décrites par J. Gracq dans La Forme d'une ville, où Nantes ne pouvait être qu'imaginée, derrière les murs étanches de l'internat (la " frontière d'alarme »); on remarque également l'analogie entre Orsenna et le texte (ce qui se vit sur les bancs d'école), et celle qui unit le Farghestan à l'oralité (au bruit ; au « cri » de A. Rimbaud, Comte de Lautréamont ou A. Jarry ; à « la rumeur de Nantes »).

8 Avant d'approfondir cette dynamique entre littératie et oralité dans Le Rivage des Syrtes, il est légitime de se demander : au-delà des législations qui font de l'apprentissage de l'écriture, une obligation nationale dans les écoles de France, y a-t-il dans le processus même qui consiste à écrire, un potentiel inévitable d'orthodoxie autoritaire? En d'autres mots, l'oppression, de même que le conservatisme en découlant (afin de maintenir intacte la domination), sont-ils affaires de textes? À ce titre, J.Goody propose, dans ses ouvrages sur les technologies de la littératie, quelques thèses des plus intéressantes sur les différences entre sociétés orales et sociétés fondées sur l'écriture lorsqu'il est question de transmission intergénérationnelle des savoirs. La société orale, celle pour qui l'écriture n'existe pas et qui n'est pas en contact avec des sociétés de l'écrit, ne possède pas les technologies propres à l'inscription de ses propres savoirs : dans ces circonstances, l'accumulation de connaissances ne peut reposer que sur la mémoire des individus vivant au sein de la communauté (Goody, 2007 [2000], p. 44). Cette situation génère par défaut une possibilité plus facile de changement : lorsque la mémoire faillit et qu'elle oublie, ou lorsque les circonstances contemporaines vont à contre-courant des savoirs traditionnels, les sociétés orales peuvent procéder avec une plus grande aisance aux changements qui leur conviennent le mieux, nonobstant des dispositions prises antérieurement. L'adaptation homéostatique constante caractérise donc les cultures orales. C'est le fait de ne pas posséder l'écriture qui les met de facto dans un état de création continuelle (le plus souvent cyclique), en raison des limites de la mémoire, du rôle de l'oubli et de l'utilisation inventive du langage et de la gestuelle (ibid., p. 78). Leur rapport au savoir, par défaut, n'est pas rigide et orthodoxe.

Du côté des sociétés de l'écriture en revanche, la tradition et la loi se trouvent consignées par écrit. Le changement et l'adaptation à des situations labiles deviennent alors plus problématiques : une fois objets d'écriture, les textes de loi et de tradition ne 
sont plus aussi adaptables que leur « contrepartie » des sociétés orales; ils nécessitent des réformes délibérées (ibid., p. 44). La stabilité du texte - et son orthodoxie - sont une conséquence directe de la trace matérielle qu'il laisse :

La mémoire orale, comme nous sommes prompts à le reconnaitre chez les autres, mais pas toujours chez nous-mêmes, peut être traîtresse, et la remémoration est souvent sélective, souvent influencée par des pressions individuelles et sociales. Il peut en être de même des conservateurs, qui sélectionnent tel ou tel document, et agissent en gardiens du temple de l'histoire, mais les documents eux-mêmes ont une existence matérielle en dehors de l'individu, existence très différente de celle $\mathrm{du}$ souvenir de la parole ; la trace est d'un autre ordre. (ibid., p. 172-173)

10 L'écriture permet l'existence de textes et de documents auxquels on peut se référer, alors que cette référence dans les sociétés orales, qui ne peuvent compter que sur la mémoire, est beaucoup moins aisée. Même si certaines nuances s'imposent, écrire amène donc un conservatisme qui n'est pas envisageable dans les sociétés qui ne connaissent pas l'écriture. Cette dichotomie entre sociétés orales et sociétés de l'écriture fait remarquablement écho à celle qui se joue entre le Farghestan et Orsenna - cette dernière étant présenté comme république pétrifiée dans ses traditions, dans " [s]a complaisance, sa sagesse, son confort, son sommeil », « son assoupissement sans âge » (Gracq, 1989c [1951], p. 641, 750).

11 L'appui de cette " complaisance » et de cette «sagesse » sur les textes, dans Le Rivage des Syrtes, est indéniable: c'est par écrit que se font toutes les procédures administratives et légales sur lesquelles repose la stabilité d'Orsenna. À titre d'exemples, un décret du Sénat confirme Aldo dans ses fonctions d'Observateur auprès des Forces Légères (ibid., p. 558); contre les bruits qui essaiment à Maremma, le même Aldo recommande la publication par Belsenza d'un « démenti officiel » (ibid., p. 634); ce sont les "pièces officielles relatives à [s]a nomination", et plus spécifiquement "l'Instruction spéciale d'Urgence ", qui le (ré)informent (de même que le lecteur) de la guerre opposant Orsenna au Farghestan(ibid., p. 559-560); les correspondances officielles sont représentées par le rapport qu'il écrit à la Seigneurie (ibid., p. 653-654) et la réponse que lui fait le Conseil de Surveillance (ibid., p. 668-675); un mot de Marino donne à Aldo la charge du navire le Redoutable pendant son absence (ibid., p. 713-714); un rapport de police lui apprend l'arrivée de l'armée farghienne à proximité d'Orsenna (ibid., p. 836-837) ; c'est, finalement, une ordonnance « rédigée et scellée » par Danielo qui proclame « l'état de siège » closant le roman (ibid., p. 838). Nous remarquons ici que non seulement l'écriture est affaire de conformisme et de tradition, au sens où c'est elle qui maintient l'ordre (ou remet en ordre les situations potentiellement chaotiques), mais elle est également affaire d'autorité, et donc de pouvoir. Pour J. Goody (2007 [2000], p. 189-190), lorsqu'elle est utilisée par un groupe déjà dominant, les techniques liées à la littératie participent effectivement à assoir et à renforcer la domination. À cet égard, dès l'Égypte ancienne, "les positions qu'occupaient les membres de l'élite étaient à la mesure des rapports qu'ils entretenaient avec l'écriture » (Goody, 1986, p. 124).

12 Toutes les figures d'autorité du roman - le narrateur (l'observateur au service de la république), Marino (le capitaine de l'Amirauté), Belsenza (le policier et enquêteur) et Danielo (un puissant politicien) principalement - sont invariablement présentés comme des êtres maniant la plume. Le travail de Marino est, rappelons-le, celui d'un comptable, d'un fonctionnaire; il pose sa pipe «sur une pile de dossiers", sa « main lente et appliquée de laboureur festonn[e] d'une encre, au travers des pages, le sillon 
quotidien» (Gracq, 1989c [1951], p.588); même dans la détresse d'un mauvais pressentiment, « [l]e travail continu[e] apparemment comme de coutume, et la pile de papiers rangée le matin à sa gauche et reformée le soir à sa droite, comme on renverse un sablier, rest[e] la figure même du temps sans secousses de ces journées de l'Amirauté » (ibid., p. 659). Le capitaine de l'Amirauté est aussi un lecteur : il possède dans sa chambre tous les ouvrages sur le Farghestan (ibid., p. 607) et dans sa cabine les volumes des Instructions nautiques (ibid., p. 726). Le policier Belsenza, quant à lui, « travaill[e] dans [un] bureau [...] à l'odeur assoiffante de papier surchauffé », fait lire à Aldo "[d]es rapports sans mot dire, le sourcil encore froncé de sa lecture » (ibid., p. 687-688), fait parvenir à Orsenna un rapport, "rédigé avec une prudence et une réticence extrêmes ", pour exposer la situation de Maremma (ibid., p. 674). Les agents de l'ordre, tout comme Marino, s'occupent également de mathématiques et de comptabilité : on dit que "[1]es statistiques de police portaient de jour en jour le témoignage d'un relâchement bizarre de la moralité, et tout particulièrement les cas d'exhibitionnisme et de provocation à la débauche» (ibid., p. 688). En tant qu'Observateur, avant d'être celui par qui passe la transgression, Aldo est un universitaire (ibid., p. 555, 595). Son parcours scolaire pourrait difficilement être plus lié à l'écriture : il est passé par «l'école de Droit diplomatique » (ibid., p. 669). Quant à Danielo, le puissant fonctionnaire et politicien de la république d'Orsenna, c'est incontestablement un être d'écriture et de papier :

Le trait le plus remarquable de sa carrière était qu'orienté dès sa jeunesse vers des recherches purement désintéressés et spéculatives (il était l'auteur d'une Histoire des origines qui faisait autorité à Orsenna pour tout ce qui concernait la période de fondation), la soixantaine passée il avait commencé à se mêler aux intrigues politiques de la ville, à l'âge où les hommes d'État sur le retour cherchent plutôt une justification de leur action passée à travers une biographie d'Agathocle ou de Marc-Antoine; et le préjugé porté contre un homme d'étude avait longtemps prévalu, jusqu'à freiner un peu même à Orsenna cette seconde carrière, contre les preuves d'opiniâtreté et de volonté incisive qu'il n'avait pas tardé à y fournir. Son caractère ombrageux lui valait peu d'amis; en dehors des heures où le service d'État l'appelait à la ville, il passait pour vivre presque seul dans sa campagne de Bordegha, au milieu de sa bibliothèque (ibid., p. 818-819).

Si l'écriture, de même que ceux qui la maîtrisent, sont associés à l'autorité dans $L e$ Rivage des Syrtes, cela ne veut pas dire que le roman valorise l'usage de la littératie et l'exercice du pouvoir pour autant. Au contraire : se faisant le récit d'une décadence, de l'implosion d'une république traditionnaliste (la narration est un souvenir qui s'écrit sur le « rougeoiement de [1]a patrie détruite » [ibid., p. 729]), le récit de J. Gracq semble plutôt se faire critique des outils par lesquels s'exercent l'oppression et la reproduction du même. Cette critique se manifeste principalement par la représentation d'un contrepouvoir, porté par la mise en scène d'une conception singulière de l'oralité. Parce qu'il ne passe pas par le texte, ce contre-pouvoir de l'oralité échappe dans Le Rivage des Syrtes au contrôle efficace de ceux qui manient la plume.

\section{D'une oralité barbare et révolutionnaire}

Orsenna fait donc sans contredit partie des sociétés de l'écriture et de la littératie. L'organisation de l'État, avec ses hiérarchies complexes, son fonctionnariat et sa comptabilité, suppose nécessairement la maîtrise d'une écriture : en témoignent tous les rapports, tous les textes de savoir, toutes les correspondances administratives qui 
sont mis en scène dans Le Rivage des Syrtes. Inversement, le Farghestan, dans son altérité ardemment désirée par la narration, se trouve dès sa première évocation, ramené au pôle contraire, celui de «l'oralité ».

On sait peu de chose dans la Seigneurie sur le Farghestan, qui fait face aux territoires d'Orsenna par-delà la mer des Syrtes. Les invasions qui l'ont balayé de façon presque continue depuis les temps antiques - en dernier lieu l'invasion mongole - font de sa population un sable mouvant, où chaque vague à peine formée s'est vue recouverte et effacée par une autre, de sa civilisation une mosaïque barbare, où le raffinement extrême de l'Orient côtoie la sauvagerie des nomades. Sur cette base mal raffermie, la vie politique s'est développée à la manière de pulsations aussi brutales que déconcertantes: tantôt le pays, en proie aux dissensions, s'affaisse sur lui-même et semble prêt à s'émietter en clans féodaux opposés par des haines de race mortelles - tantôt une vague mystique, née dans le creux de ses déserts, fond ensemble toutes les passions pour faire un moment du Farghestan une torche aux mains d'un conquérant ambitieux (ibid., p. 560).

Face au confort, à la stabilité et à la vieillesse d'Orsenna, le Farghestan, «balayé », rasé "de façon presque continue», apparaît comme une société qui, n'ayant jamais la possibilité de construire sur des assisses solides, resterait toujours jeune (dans l'enfance d'une civilisation pour reprendre l'Oswald Spengler du Déclin de l'occident ayant inspiré J. Gracq1), constamment soumise aux origines desquelles elle n'arriverait pas à émerger. Certaines formules nous permettent déjà de faire des assomptions sur le rapport qu'entretiendrait le Farghestan avec l'écriture - dans ce qui distingue ce rapport de celui qu'entretient Orsenna. Même s'il est vrai, avec cette brève description du Farghestan, que nous sommes loin du changement homéostatique, de l'adaptation graduelle aux circonstances que connaitraient les sociétés orales, plusieurs éléments viennent néanmoins radicalement l'opposer au pays d'écriture d'où émerge l'énonciation d'Aldo. Le Farghestan se positionne en effet, au-delà de son éternelle jeunesse, en tant que parfait antagoniste d'Orsenna : il est « oriental », « mongol », une " mosaïque barbare » qui répond à ce que lui dictent ses haines et ses passions, qui a la "sauvagerie des nomades». C'est le mouvement (les vagues, l'affaissement et l'émiettement) et l'instabilité (une «base mal raffermie ») qui le qualifient, faisant de "sa population un sable mouvant", de sa politique un ensemble de "pulsations aussi brutales que déconcertantes ». Contraires à ceux d'Orsenna, les traits distinctifs du Farghestan sont également incompatibles avec ceux qui rendraient possible et que favoriseraient du même coup l'écriture: la barbarie, la sauvagerie, l'instabilité, le mouvement continuel s'opposent à l'orthodoxie, à l'immuabilité, au conservatisme, à la stabilité, à la rigidité et à la résistance au changement. Pas étonnant, finalement, qu'on dise savoir "peu de chose dans la Seigneurie sur le Farghestan» lorsqu'il est mentionné que «chaque vague à peine formée [se voit] recouverte et effacée par une autre »: si l'écriture existe bel et bien au Farghestan (encore que cette façon d'exister n'est peut-être pas tout à fait assumée par le récit $^{2}$ ), elle ne prend visiblement pas en charge efficacement sa fonction d'enregistrement et de transmission - entre nations et entre générations - des savoirs.

16 Jamais nous ne lisons sur le Farghestan de " première main ${ }^{3}$ ", c'est-à-dire à travers un personnage qui en viendrait directement (en ce qui concerne «l'Envoyé », voir note 2). De « seconde main » seulement donc, lorsque le discours sur la nation ennemie advient, c'est toujours d'une manière qui n'est pas posée sur le papier. Il est même explicitement dit qu'il serait impossible d'inscrire cette information dans un texte - ce qui suggère l'idée que le Farghestan est une société où l'oralité joue un rôle 
prépondérant. Lorsqu'on réfère au Farghestan (la référence est faite, systématiquement, de manière indirecte: "[o]n ne parle au fond guère " va même jusqu'à affirmer le policier Belsenza [ibid., p. 634]), « [c]e sont des bruits » (ibid., p. 631) dont il est « impossible de retracer l'origine » (ibid., p. 632), impossible à vérifier (ibid., p. 634), « des bruits incontrôlables » (ibid., p. 761) ; ce sont « des bruits qui courent dans la ville» (ibid., p. 758), une rumeur qu'il n'est pas possible de consigner par écrit (ibid., p. 634). Le Farghestan apparaît dans le discours d'une façon évanescente, au sein de bruits qui disparaissent sans laisser aucune trace de leur passage - au contraire d'un texte qu'il est possible de relire, de vérifier et de contre-vérifier étant donné sa stabilité dans l'espace et dans le temps. Belsenza, expliquant au narrateur la situation difficile du corps policier de faire face à ces bruits, résume à l'aide de sa métaphore les principales caractéristiques qui leur sont associées: leur insaisissabilité, leur mouvement perpétuel ainsi que l'absence de traces qu'ils laissent derrière eux.

Tout renvoie aux bruits, mais rien ne les dénonce. Comme si les paroles, toutes les paroles d'une journée, dessinaient obstinément un moule - le moule de quelque chose -, mais que ce moule restât vide. Je me fais bien mal comprendre. Je vais me servir encore d'une image. Vous connaissez le jeu du furet. Tout le monde fait cercle, les mains sont fermées sur la corde, on ne voit rien, mais les mains sont complices, le furet court, glisse le long de la corde, repasse, tourne inlassablement. Il n'est jamais là. Chaque main est vide, mais chaque main est un creux tiède pour l'accueillir, pour l'avoir accueilli. Voilà à quel jeu joue Maremma toute la journée. Et je ne suis pas tout à fait sûr que ce soit un jeu. (ibid., pp. 634-635)

Le Rivage des Syrtes se construit ainsi comme le graduel envahissement d'une société écrite par l'oralité d'une société barbare, envahissement qui s'inscrit principalement dans la transformation radicale de la nature du lien social. L'écriture, associée à la domination et à l'autorité, hiérarchise - et donc divise - des groupes, atomisant les individus dans un champ d'actions et de discours adapté à leur position sociale. Sa mise à mal par le contre-pouvoir de l'oralité, qui mène ultimement à la destruction d'Orsenna, passe donc par un singulier communautarisme. Effectivement, les «bruits », lorsqu'ils envahissent Orsenna, brisent les rapports traditionnels de domination en fabriquant de nouveaux liens; ils créent une fraternité horizontale, bien différente de la verticalité des rapports de l'ancienne république. Les bruits instaurent autour d'eux une communauté hétérogène et disparate qui rassemble « des gens qui ne se fussent jamais salués à Orsenna » (ibid., p. 627). Ils égalisent, brisent les hiérarchies, mélangent les individus d'une façon qui évoque «le cousinage spontané, la franc-maçonnerie intime des villes d'eaux où l'on vient soigner une maladie grave ", ils parviennent à faire d'une assistance une foule fusionnelle et indifférenciée (ibid., p. 627, 707-712).

$[\mathrm{P}]$ ar-delà les différences de classe et de richesse, cette espèce de fraternité spontanée de la rue ressemblait à celle de gens embarqués dans le même bateau, liés par la solidarité des réflexes d'un équipage de navire au moment où il appareille et où les mots de « mort » ou de « maladie » s'éteignent dans l'imagination au profit de ceux de «typon» ou de «naufrage». Un grand privilège partagé détendait les ressorts de la jalousie et de l'envie, égalisait les rangs et brassait les remous d'une masse devenue plus fusible: celui d'un peuple entier, collé au sol et maintenant averti par son oreille profonde, que les temps venus poussaient sur la scène, et qui pêle-mêle, abandonnant ses venelles et ses caves, se bousculait d'instinct dans le désordre vers le seul jour qui vaille qu'on s'y brûle : le grand jour (RS, p. 815).

Brisant les hiérarchies et les relations traditionnelles entre dominants et dominés qui prévalent au départ dans Le Rivage des Syrtes, l'oralité se trouve peu à peu à occuper dans le roman des positions qui ne lui sont pas à priori naturelles : celles des vainqueurs 
et des dominants. Cependant, quelque chose fait tache chez J. Gracq dans cette représentation d'une lutte entre écriture et oralité, et cette « tache » a tout à voir avec ce que nous avons appelé poétique de l'internat. Elle est inhérente au paradoxe dans lequel on s'inscrit lorsqu'on écrit contre les murs - ce paradoxe qui rend absolument nécessaire la surface qu'on cherche à détruire par le récit.

\title{
L'ambivalence contre-révolutionnaire d'une poétique de l'internat
}

19 Comme toutes les autres fictions gracquiennes, Le Rivage des Syrtes se termine au seuil de l'événement (la guerre contre le Farghestan) qu'annonçait pourtant la totalité du récit. L'événement, chez l'écrivain, est toujours extérieur au cadre de la représentation. Michel Murat a montré combien la présence d'indices sur l'inévitable fin peut bien se faire sentir chez J. Gracq sans que cela n'empêche la narration d'être amputée d'une conclusion, d'une résolution au sens plein du terme.

\begin{abstract}
Ainsi le livre "tourne à la rature de l'événement » : c'est un mot de Gracq que l'on cite souvent. Mais on en retient l'aveu d'une déception et d'un manque, sans voir comment l'écrivain fait nécessité de vertu. L'événement est à la fois vertige et spectacle. Impossible à raconter, il s'immobilise dans des instantanés qui le représentent de manière indirecte, diffractée : tableaux ou gravures, mascarades qui recourent à la médiation explicite d'une œuvre d'art, scènes oniriques; à la limite, tout objet que le regard met en scène (comme la forteresse ou les ruines de Sagra) ou reconstruit dans l'obsession de la mémoire (Heide violée). (Murat, 2004, p. 87-88)
\end{abstract}

En ce sens, la littérature de J. Gracq correspondrait à une «liturgie du désir » (Le Guillou, 1991, p. 76). Ce serait, en d'autres mots, une « littérature de l'attente», « une littérature du dilatoire comme l'est un texte pornographique» (Biasi, Michon, 2007, p. 35). En tant que telle, sa littérature en serait une d'avant les ébats, en serait une des "préliminaires »: «[C]ette attente, dont le récit nous force à partager l'exaltation, ressemble beaucoup à des préludes amoureux : or, précisément, ce qui intellectualise vertigineusement le rituel érotique, ce n'est pas l'acte, c'est le préliminaire » (ibid.). On parvient donc souvent à la conclusion, quand on analyse la littérature de J. Gracq, qu'elle est en quelque sorte, une littérature de prélude : «[L]e récit forme un prélude à une représentation qui n'aura pas lieu, qui ne pourra pas même avoir lieu après, pour la raison que le récit est cette représentation du prélude » (Vouilloux, 1989, p. 323).

$21 \mathrm{Au}$ regard des fragments que J. Gracq consacre à l'expérience de l'internat, celle d'écrivains aimés comme la sienne propre, une autre interprétation de cette «littérature du désir» est possible - interprétation qui poserait les bases d'une poétique de l'internat. L'enfermement et l'oppression invitent l'écrivain à la représentation d'un ailleurs libre et révolutionnaire ; ils constituent donc les conditions nécessaires à l'écriture. Contre l'enfermement et l'oppression, le récit devient cette possibilité d'un monde altéré, radicalement différent de celui qu'il nous est donné d'expérimenter. Or, pour J.Gracq, l'actualisation d'une authentique révolution, révolution qui détruirait les rapports de domination à l'origine de l'enfermement et de l'oppression, briserait les conditions de l'imagination des internes. Si le désir révolutionnaire ne trouve jamais résolution dans Le Rivage des Syrtes (comme dans les autres fictions gracquiennes), si la transgression y est toujours synonyme d'impossibilité de mise en récit, c'est que l'oppression est le terreau nécessaire au 
fonctionnement de l'imagination. La liberté doit demeurer hors texte; elle ne peut rester qu'un impossible.

Cette dialectique indépassable de la fiction gracquienne se manifeste également dans le rapport thématique que Le Rivage des Syrtes entretient avec l'écriture. Il faut à cet égard mettre en lumière que la lutte des «bruits » contre les « textes ", du Farghestan contre Orsenna, ne trouve pas son origine dans la décision d'une nation ennemie (fondée sur l'oralité) d'entrer en guerre. Cette guerre est en fait déjà gravée dans un texte de loi, et c'est l'absence de rectification, l'absence d' "envie de terminer légalement le conflit " (Gracq, 1989c [1951], p.561), qui la laisse latente et lui laisse son potentiel de réactivation. Les paroles sont évanescentes, il suffit de penser à autre chose pour qu' " un souffle brusque » vienne dissiper «les nuées d'orage » qu'elles représentent (ibid., p. 594) mais l'écriture, elle, crée de "l'irréparable » (ibid., p. 653) : pour revenir aux thèses de J. Goody, c'est le texte qui crée l'orthodoxie (et qui maintient intactes les guerres vieilles de trois cents ans). La "nouvelle " guerre contre le Farghestan a beau avoir des circonstances particulières et des «héros " différents, elle ne constitue au fond que la réactivation d'une guerre originelle déjà inscrite dans le papier. Elle n'est, au fond, qu'une interprétation d'un même texte -lui-même resté identique. Le mouvement du Rivage des Syrtes suit peut-être la pente d'un passage graduel de l'écriture à l'oralité, de la "civilisation " d'Orsenna à la "barbarie » du Farghestan: cependant, la guerre elle-même, noyau du récit, renvoie au primat de l'écriture, renvoie au caractère indépassable de ce qui a été inscrit et qui n'a pas été corrigé dans les textes. Une fois quelque chose consignée par écrit, il n'est pas possible de revenir en arrière, il faut absolument écrire par-dessus. Le mouvement du roman par rapport à cette lutte entre littératie et oralité est donc paradoxal. La seule échappatoire possible à une société écrite se trouverait, ultimement, déjà imprimée dans les textes.

Pour conclure, considérons un instant Le Rivage des Syrtes lui-même en tant que texte : à cet égard, l'envahissement des bruits dans le roman apparaît désormais artificiel, en ce sens que l'oralité contaminatrice se trouve elle-même mise en scène au sein de et par l'écriture. Malgré la liberté qu'entretient J. Gracq avec l'orthodoxie du langage (Borgal, 1993, p. 213), voire de son utilisation fautive du français écrit (Boisdeffre, 1964 [1944], p. 362), Le Rivage des Syrtes comme roman est en lui-même un produit de l'écriture et il ne peut y avoir de véritable oralité qu'hors texte. C'est là, également, que se manifeste le primat de l'écriture. Seules les confessions écrites d'Aldo laisseront une empreinte. Tout ce qui en sortira sera nécessairement évacué de l'histoire. Si l'on revient aux traces que laisse l'internat dans la poétique de J. Gracq, on en arrive à une troublante contradiction. Pour pouvoir témoigner dans son œuvre des blessures laissées par le régime des internes, J. Gracq n'a, en tant qu'écrivain, d'autre choix que d'utiliser l'enseignement sur lequel s'inscrivent les contusions de sa dureté autoritaire: l'écriture. 


\section{BIBLIOGRAPHIE}

BIASI, P. (de) \& MICHON, P. (2007). « Une littérature de l'attente ». Le Magazine littéraire 465, p. 34-38.

BOIE, B. (1966). Hauptmotive im Werke Julien Gracqs. Munich : W. Fink.

BOISDEFFRE, P. (1964) [1944]. Histoire vivante de la littérature d'aujourd'hui. Paris : Perrin.

BORGAL, C. (1993). Julien Gracq. L'écrivain et les sortilèges. Paris : Presses universitaires de France.

FOUCAULT, M. (2013). La Société punitive. Cours au Collège de France, 1972-1973. Paris : EHESS/

Gallimard/Seuil.

FURET, F. \& OZOUF, J. (dirs) (1977). Lire et écrire. L'alphabétisation des Français de Calvin à Jules Ferry.

Paris : Minuit.

GRACQ, J. (1989a). « Chronologie ». In : Cuvres complètes I. Paris : Gallimard, p. LIX-LXXXII.

GRACQ, J. (1989b) [1961]. « Préférences ». In : Cuuvres complètes I. Paris : Gallimard, p. 841-1000.

GRACQ, J. (1989c) [1951]. « Le Rivage des Syrtes ». In : Ceuvres complètes I. Paris : Gallimard, p. 553-839.

GRACQ, J. (1995) [1985]. « La Forme d'une ville ». In : Cuvres complètes II. Paris : Gallimard, p. 769-878.

GRACQ, J. (2002) [1981]. Entretiens. Paris : José Corti.

GOODY, J. (1986). La Logique de l'écriture. Aux origines des sociétés humaines. Trad. de l'anglais par A.M. Roussel. Paris : Armand Colin.

GOODY, J. (2007) [2000]. Pouvoirs et savoirs de l'écrit. Trad. de l'anglais par C. Maniez. Paris : La Dispute.

GOODY, J. (2014) [2010]. Mythe, rite \& oralité. Trad. de l'anglais par C. Maniez. Nancy : Presses universitaires de Nancy.

LE GUILLOU, P. (1991). Julien Gracq. Fragments d'un visage scriptural. Paris : La Table Ronde.

MAYEUR, F. (2004) [1981]. Histoire de l'enseignement et de l'éducation III. 1789-1930. Paris : Perrin.

MURAT, M. (2004). L'enchanteur réticent. Essai sur Julien Gracq. Paris : José Corti.

vouilloux, B. (1989). De la peinture au texte. L'image dans l'œuvre de Julien Gracq. Genève : Droz.

\section{NOTES}

1. " J'ai dû lire Le Déclin de l'Occident vers 1946 ou 47, sans doute au moment où j'écrivais Le Rivage des Syrtes. [...] Je crois que la lecture de Spengler, qui a été pour moi passionnante, (je considère d'ailleurs Spengler comme un poète de l'histoire plutôt qu'un philosophe) a autorisé pour moi et un peu légitimé l'accent que je mettais depuis longtemps sur certaines périodes de l'histoire qui me parlent plus que d'autre : je veux dire les périodes de décadence : celles où une civilisation s'endort et meurt toute seule - sans avoir besoin de chocs extérieurs - on pourrait dire de vieillesse » (Boie, 1966, p. 197). 
2. Il existerait une Chancellerie de Rhages, et donc des papiers officiels du Farghestan : " Je dépliai le papier qu'il me tendait, et soudain mes yeux devinrent fixes. À l'angle de droite, portant le serpent entrelacé à la chimère, et tel que je l'avais si souvent déchiffré à l'Académie diplomatique au bas de traités poussiéreux et centenaires, le sceau de la Chancellerie de Rhages étoilait la feuille. Le texte certifiait le caractère pacifique de la mission du porteur et, en l'accréditant, priait expressément qu'on lui accordât les égards et le traitement officiel réservés aux parlementaires de guerre " (Gracq, 1989c [1951], p. 752-753). Malgré cela, il est intéressant de constater que « l'Envoyé » ne « vient » pas directement du Farghestan : il fait partie de la « livrée de la princesse Aldobrandi » (ibid., p. 753), il a appris la langue du pays (ibid., p. 754), il a vécu à Orsenna assez longtemps pour «l'aimer" (ibid., p. 761), il a une "manière impersonnelle » de parler de l'autorité qu'il représente (ibid., p. 756). Narrativement, il est le double négatif d'Aldo qui vient lui donner confirmation de son acte et suggère son caractère irrémédiable; il ne pourrait être qu'une « hallucination » (ibid., p. 764) ou bien qu'un rêve, son arrivée et sa sortie étant étrangement symétriques (ibid., p. 752, 763). Aucune autre manifestation de l'écriture farghienne moins ambiguë n'apparaît d'ailleurs dans le récit.

3. Ce statut particulier de l'« oralité » dans Le Rivage des Syrtes explique en grande partie la raison pour laquelle sa définition y semble toujours être négative (ce qui ne saurait apparaître dans les textes) - ce qui bien entendu se reflète dans cet article. Il fait singulièrement écho à la manière dont la «tradition orale » se trouve circonscrite et influencée par l'écriture (Goody, 2014 [2010], 41-59).

\section{RÉSUMÉS}

Dans cet article, je me propose d'étudier une articulation singulière se jouant au sein de l'écriture du déclin dans Le Rivage des Syrtes de Julien Gracq (1989c [1951]) : la dialectique entre littératie et oralité. Si la maîtrise de l'écriture et de la lecture est associée au pouvoir dans le roman, et qu'à l'inverse, l'oralité semble vouée à l'impossibilité d'une véritable institutionnalisation, le récit nous fait la démonstration ambivalente d'une certaine conquête de la littératie par les «bruits » et la rumeur ambiante - par ce qui, finalement, n'est jamais posé sur le papier. Utilisant des outils de l'ethnocritique, j'analyse la manière dont l'opposition entre littératie et oralité se résout chez J. Gracq par un étonnant paradoxe: mettre en récit la dégénérescence de la littératie, c'est encore utiliser l'acte dont le mouvement du texte propose l'abolition - l'écriture.

In this article, I intend to explore a singular relationship playing within the writing of decline in Julien Gracq's (1989c [1951]) The Opposing Shore: the dialectic between literacy and orality. While a command of writing and reading is associated with power in the novel, whereas orality appears destined to forever resist true institutionalization, the story offers an ambivalent depiction of a certain conquest of literacy by "noise" and rumours -in other words, by elements that are never committed to paper. By using an ethnocritical approach, I will analyze the way in which the opposition between literacy and orality in J. Gracq's novel is resolved through a surprising paradox: recounting the degeneration of literacy is only possible through the very act that the text proposes to abolish - the act of writing. 
INDEX

Mots-clés : littérature, ethnocritique, Julien Gracq, le Rivage des Syrtes, oralité, littératie

Keywords : literature, ethnocriticism, Julien Gracq, The Opposing Shore, Orality, Literacy

\section{AUTEUR}

\section{ÉMILE BORDELEAU-PITRE}

Université du Québec à Montréal, C-H2L 2C4 Montréal, Cananda 